\title{
On some properties of $\beta$-Laplace integral transform
}

\author{
Garima Agarwal $^{\mathrm{a}}$, Amit Gaur ${ }^{\mathrm{a}}$, Kottakkaran Sooppy Nisar ${ }^{\mathrm{b}, *}$, Abdallah Hassan Abusufian ${ }^{\mathrm{b}}$ \\ ${ }^{a}$ Department of Mathematics and Statistics, Manipal University Jaipur, Rajasthan, India. \\ ${ }^{b}$ Department of Mathematics, College of Arts and Sciences, Wadi Aldawaser, Prince Sattam bin Abdulaziz University, Saudi Arabia.
}

\begin{abstract}
This paper aims to discuss and resolve some shortcomings of the classical Laplace integral transform of a particular class of functions such as poles (if exist) lie on fixed points, fixed s-domain, and analyticity region for any function of that particular class of functions. We mainly try to solve these shortcomings by employing a new generalized Laplace transform named the $\beta$-Laplace integral transform.
\end{abstract}

Keywords: Laplace integral transform, $\beta$-Laplace integral transform, s-domain, analytic function.

2020 MSC: 44A10, 44A15.

(C)2021 All rights reserved.

\section{Introduction}

An integral transform is any transform $\mathrm{T}$ of the following form [4]

$$
T(f)_{(s)}=\int_{t_{1}}^{t_{2}} K(t, s) f(t) d t
$$

Here, $f(t)$ is input, $T(f)_{(s)}$ is output function with a new parameter $s$ independent of $t$ and $K(t, s)$ is called the kernel of integral transform. Mainly integral transform is a function from its original domain into another more suitable domain where it might be solved much more easily than its previous domain. Thereafter, the solution is transformed back to the original domain using the inverse of that integral transform [22].

The Laplace integral transform [18] is an old, famous, and widely used integral transform than other integral transforms. The Laplace transform plays a vital role in solving engineering sciences problems $[2,5,6,11,20,23]$.

In recent past, many new integral transforms have been introduced, for instance, Sumudu, Kamal, Mohand, Natural, Polynomial, Sawi, ZZ-transform, Sadik, Tarig, Aboodh, Mahgoub, AF Transform. These

\footnotetext{
${ }^{*}$ Corresponding author

Email addresses: garima.agarwal@jaipur .manipal .edu (Garima Agarwal), gaur84amit@gmail.com (Amit Gaur), n. sooppy@psau.edu.sa (Kottakkaran Sooppy Nisar), sufianmath97@hotmail.com (Abdallah Hassan Abusufian)

doi: $10.22436 /$ jmcs.023.04.04
}

Received: 2020-09-17 Revised: 2020-10-16 Accepted: 2020-10-22 
all-new transforms are commonly using the exponential type kernel, and they are a generalized form of the Laplace transform $[1,3,7,8,12-16,21,24,26]$.

But, recently Gaur et. al. [10] has introduced a very new form of generalized Laplace integral transform with a new parameter $\beta>1$ and defined as

$$
\mathcal{L}_{\beta}\{f(t)\}_{(s)}=\int_{0}^{\infty} \beta^{-s t} f(t) d t, \quad \beta>1 .
$$

Every function $f(t), t \geqslant 0$ of exponential order and sectionally continuous on $[0, \infty)$ is called a function of class A. It is a sufficient condition for existence of the Laplace and the $\beta$-Laplace integral transform that a function $f$ is of class A.

On the function of class A, the Laplace transform has many disadvantage as poles of a class A function (if exists) lie at a fixed points, constant s-domain $(\operatorname{Re}(s)>\alpha$ ), therefore these are drawback of the Laplace transform which restrict to its full applicability in many fields of basic science and engineering such as signal processing, digital communication, electrical engineering, control theory, medical science $[9,17,19$, 25].

This is the motivation of our paper to find the solution of above-mentioned problems. On this class A, The $\beta$-Laplace integral transform has many advantages over the Laplace integral transform such as variable s-domain, duality with the Laplace transform, shifting of poles, variable domain of analyticity, necessary condition for existence of the $\beta$-Laplace integral transform, which is also can be applied on the Laplace integral transform.

\section{Main results}

Theorem 2.1 (Expansion and compression of $s$-domain). For a function $f(t), t \geqslant 0$ which is a function of class $A$,

(i) if $\beta>e$, then $(\mathrm{SD})_{\mathrm{L}} \subset(\mathrm{SD})_{\mathrm{L}_{\beta}}$;

(ii) if $\beta>e$, then $(\mathrm{SD})_{\mathrm{L}} \subset(\mathrm{SD})_{\mathrm{L}_{\beta}}$,

where $(\mathrm{SD})_{\mathrm{L}}$ and $(\mathrm{SD})_{\mathrm{L}_{\beta}}$ are s-domain of the Laplace and the $\beta$-Laplace integral transform of function $f \in A$, respectively.

Proof. Firstly $f$ is of exponential order therefore there exist $M_{1}>0, \alpha>0$ and $t_{0} \geqslant 0$ such that

$$
|f(t)| \leqslant M_{1} e^{\alpha t}, \quad t \geqslant t_{0} .
$$

Also, $f(t)$ is sectionally continuous on $\left[0, t_{0}\right]$ and hence bounded, so there exists $M_{2}$, say

$$
|f(t)| \leqslant M_{2}, \quad 0<t \leqslant t_{0} .
$$

Since $e^{\alpha t}$ has a positive minimum on $\left[0, t_{0}\right]$, a constant $M$ can be chosen sufficiently large so that

$$
|f(t)| \leqslant M e^{\alpha t}, \quad t \geqslant 0 .
$$

Therefore,

$$
\int_{0}^{\tau}\left|\beta^{-s t} f(t)\right| d t \leqslant M \int_{0}^{\tau} e^{-(\xi \log \beta-\alpha) t} d t=\left\{\frac{M e^{-(\xi \log \beta-\alpha) t}}{-(\xi \log \beta-\alpha)}\right\}_{0}^{\tau}=\frac{M}{(\xi \log \beta-\alpha)}-\frac{M e^{-(\xi \log \beta-\alpha) t}}{-(\xi \log \beta-\alpha)} .
$$

Letting limit $\tau \rightarrow \infty$ and noting that $\operatorname{Re}(\mathrm{s})=\xi$, yields

$$
\int_{0}^{\infty}\left|\beta^{-s t} f(t)\right| d t \leqslant \frac{M}{(\xi \log \beta-\alpha)} .
$$


Thus, the Laplace transform converges absolutely for

$$
\xi=\operatorname{Re}(s)>\frac{\alpha}{\log \beta} .
$$

Therefore, s-domain of the Laplace transform for function $f$ is

$$
(\mathrm{SD})_{\mathrm{L}}=\{s \in \mathbb{C}: \operatorname{Re}(s)>\alpha\}
$$

Similarly, we get s-domain of the $\beta$-Laplace transform for function $f$

$$
(\mathrm{SD})_{\mathrm{L}_{\beta}}=\left\{\mathrm{s} \in \mathbb{C}: \operatorname{Re}(\mathrm{s})>\frac{\alpha}{\log \beta}\right\} .
$$

Obviously, if $\beta>e$, then

$$
\log \beta>1 \Rightarrow \alpha>\frac{\alpha}{\log \beta} \Rightarrow(\mathrm{SD})_{\mathrm{L}_{\beta}} \supset(\mathrm{SD})_{\mathrm{L}}
$$

And also, if $1<\beta \leqslant e$, then

$$
\log \beta \leqslant 1 \Rightarrow \alpha \leqslant \frac{\alpha}{\log \beta} \Rightarrow(\mathrm{SD})_{\mathrm{L}_{\beta}} \subseteq(\mathrm{SD})_{\mathrm{L}}
$$

Theorem 2.2 (Duality of the Laplace and $\beta$-Laplace integral transform). A function $\mathrm{f}(\mathrm{t}), \mathrm{t} \geqslant 0$ has the Laplace integral transform if and only if $\mathrm{f}(\mathrm{t})$ has the $\beta$-Laplace integral transform.

Proof. Let the function $f(t)$ has the Laplace integral transform $\mathcal{L}\{f(t)\}_{(s)}$ then by the definition of the $\beta$-Laplace integral transform

$$
\mathcal{L}\{f(t)\}_{(s)}=\int_{0}^{\infty} e^{-s t} f(t) d t .
$$

If $\beta>1$ be any parameter then we can re-write the above equation as

$$
\mathcal{L}_{\beta}\{f(t)\}_{(s)}=\int_{0}^{\infty} \beta^{\log _{\beta} e^{-s t}} f(t) d t=\mathcal{L}_{\beta}\{f(t)\}_{(s)}=\int_{0}^{\infty} \beta^{\left(\log _{\beta} e\right) t} f(t) d t=\mathcal{L}_{\beta}\{f(t)\}_{\left(s \log _{\beta} e\right)} .
$$

Conversely let the function $f(t)$ has the $\beta$-Laplace integral transform $\mathcal{L}_{\beta}\{f(t)\}_{(s)}$ then by the definition of the $\beta$-Laplace integral transform

$$
\mathcal{L}_{\beta}\{f(t)\}_{(s)}=\int_{0}^{\infty} \beta^{-s t} f(t) d t, \quad \beta>1 .
$$

Therefore, if whenever the Laplace transform of a function exists, then $\beta$-Laplace integral transform also exists and converges.

Theorem 2.3 (Shifting of poles). The function $\mathrm{f}(\mathrm{t}), \mathrm{t} \geqslant 0$ is any function of class $A$ and if the Laplace transform has a pole at point a of order $m$ then the $\beta$-Laplace integral transform also has pole at point $\frac{a}{\log \beta}$ of order $m$, i.e., pole at point a can be shifted to other point by choosing appropriate value of $\beta$.

Proof. Let the Laplace transform of the function $\mathcal{L}\{\mathbf{f}(\mathrm{t})\}_{(\mathrm{s})}$ has pole at point SaS of order $\mathrm{m}$, then by the definition of pole

$$
\lim _{s \rightarrow a}\left|\mathcal{L}\{f(t)\}_{(s)}\right| \rightarrow \infty
$$

And we can also write as

$$
\mathcal{L}\{f(t)\}_{(s)}=\frac{\phi(s)}{(s-a)^{m}}, \text { where } \phi(a) \neq 0 .
$$


By Theorem 2.2 the $\beta$-Laplace integral transform of the function $f(t)$ will be

$$
\mathcal{L}_{\beta}\{\mathrm{f}(\mathrm{t})\}_{(\mathrm{s})}=\mathcal{L}\{\mathrm{f}(\mathrm{t})\}_{\left(\mathrm{s} \log _{e} \beta\right)}=\frac{\phi(s \log \beta)}{(\mathrm{s} \log \beta-\mathrm{a})^{\mathrm{m}}} .
$$

In this case, we get pole at point $\frac{a}{\log \beta}$, which shows that it depends on the value of the $\beta$ therefore by choosing value of $\beta$ the pole at a can be shifted to any other point.

Theorem 2.4 (Region of analyticity). Let $\mathrm{f} \in \mathrm{A}$, then $\mathcal{L}_{\beta}\{\mathrm{f}(\mathrm{t})\}_{(\mathrm{s})}$ is an analytic function in the domain $\operatorname{Re}(\mathrm{s})>$ $\frac{\alpha}{\log \beta}$ and also

(i) if $\beta>e$, then $(D A)_{L} \subset(D A)_{L_{\beta}}$;

(ii) if $1<\beta \leqslant e$, then $(D A)_{L} \supseteq(D A)_{L_{\beta}}$.

Proof. Let $f \in A$ therefore $\exists M>0, \alpha>0$ for some $t_{0} \geqslant 0$ such that

$$
|f(t)| \leqslant M e^{\alpha t}, \quad t>t_{0},
$$

for $s=x+i y$,

$$
\begin{aligned}
\mathcal{L}_{\beta}\{f(t)\}_{(s)}=\int_{0}^{\infty} \beta^{-s t} d t=\int_{0}^{\infty} \beta^{-(x+i y) t} d t & =\int_{0}^{\infty} \beta^{-x t}(\cos (y t \log \beta)-\sin (y t \log \beta)) f(t) d t \\
& =\int_{0}^{\infty} \beta^{-x t} \cos (y t \log \beta) d t \int_{0}^{\infty} \beta^{-s t} \sin (y t \log \beta) f(t) d t \\
& =\mathfrak{u}(x, y)+\mathfrak{i v}(x, y) .
\end{aligned}
$$

Now consider

$$
\begin{aligned}
\left|\int_{t_{0}}^{\infty} \frac{\partial}{\partial x}\left(\beta^{-x t} \cos (y t \log \beta)\right) f(t) d t\right| & =\left|\int_{t_{0}}^{\infty}-t \log \beta \beta^{-x t} \cos (y t \log \beta) f(t) d t\right| \\
& \leqslant \int_{t_{0}}^{\infty}(t \log \beta) \beta^{-x t}|f(t)| d t \leqslant \frac{M}{(x \log \beta-\alpha)} \beta^{-(x-\alpha) t_{0}}
\end{aligned}
$$

Then for $x \log \beta>\alpha$, implying that the integral $\int_{0}^{\infty}\left(\frac{\partial}{\partial x}\right) \beta^{-x t} \cos (y t \log \beta) f(t) d t$ converge uniformly in the region $\operatorname{Re}(s)>\frac{\alpha}{\log \beta}$.

Likewise, the integral $\int_{0}^{\infty}\left(\frac{\partial}{\partial x}\right)\left(-\beta^{-x t} \cos (y t \log \beta)\right) f(t) d t$ converges uniformly in $\operatorname{Re}(s)>\frac{\alpha}{\log \beta}$. Because of this uniform convergence, and the absolute convergence of $\mathcal{L}_{\beta}\{\mathbf{f}\}_{(s)}$, we can differentiate under the integral sign, that is to say,

$$
\begin{aligned}
& u_{x}=\int_{0}^{\infty}\left(\frac{\partial}{\partial x}\right)\left(\beta^{-x t} \cos (y t \log \beta) f(t)\right) d t=\int_{0}^{\infty}(-t \log \beta) \beta^{-x t} \cos (y t \log \beta) f(t) d t, \\
& v_{y}=\int_{0}^{\infty}\left(\frac{\partial}{\partial y}\right)\left(-\beta^{-x t} \sin (y t \log \beta) f(t)\right) d t=\int_{0}^{\infty}(-t \log \beta) \beta^{-x t} \cos (y t \log \beta) f(t) d t,
\end{aligned}
$$

and so $u_{x}=v_{y}$. In a similar fashion we can show that $u_{y}=-v_{x}$. These partial derivatives are continuous. Thus, the Cauchy-Riemann conditions [12] are satisfied and $\mathcal{L}_{\beta}\{f\}_{(s)}=\mathfrak{u}(x, y)+\mathfrak{i} v(x, y)$ is an analytic function in the domain $\operatorname{Re}(s)>\frac{\alpha}{\log \beta}$. In similar fashion as above applied on the Laplace transform, we get $\mathcal{L}_{\beta}\{f\}_{(s)}$ is an analytic function in the domain $\operatorname{Re}(s)>\alpha$. Now domain of analyticity of the Laplace transform of function $f,(D A)_{L}$, can be defined as

$$
(D A)_{L}=\{s \in \mathbb{C}: \operatorname{Re}(s)>\alpha\} .
$$


And, domain of analyticity of the $\beta$-Laplace transform of function $f,(D A)_{L_{\beta}}$, can be defined as

$$
(D A)_{L_{\beta}}=\left\{s \in \mathbb{C}: \operatorname{Re}(s)>\frac{\alpha}{\log \beta}\right\} .
$$

Obviously, if $\beta>e$, then $(D A)_{L} \subset(D A)_{L_{\beta}}$, and also, if $1<\beta \leqslant e$, then $(D A)_{L} \supseteq(D A)_{L_{\beta}}$.

Theorem 2.5 (Necessary conditions of existence of $\beta$-Laplace integral transform of class $A$ function). Let $\mathrm{f} \in \mathrm{A}$ and $\mathcal{L}_{\beta}\{\mathrm{f}(\mathrm{t})\}_{(\mathrm{s})}$ be the $\beta$-Laplace integral transform of function $\mathrm{f}$, then it satisfies

$$
\left\{s \frac{\partial}{\partial s}-\beta \log \beta \frac{\partial}{\partial \beta}\right\} \mathcal{L}\{f(t)\}_{(s)}=0,
$$

or,

$$
\mathcal{T} \mathcal{L}\{f(t)\}_{(s)}=0, \text { where } \mathcal{T} \equiv\left\{s \frac{\partial}{\partial s}-\beta \log \beta \frac{\partial}{\partial \beta}\right\} .
$$

By the definition of the $\beta$-Laplace integral transform

$$
\mathcal{L}_{\beta}\{f(t)\}_{(s)}=\int_{0}^{\infty} \beta^{-s t} f(t) d t, \quad \beta>1 .
$$

By Theorem 2.4, if $\mathrm{f} \in \mathrm{A}$, then $\mathcal{L}_{\beta}\{\mathrm{f}(\mathrm{t})\}_{(\mathrm{s})}$ is analytic in the domain $\operatorname{Re}(\mathrm{s})>\frac{\alpha}{\log \beta}$. Partial differentiating with respect to $\mathrm{s}$, we obtain

$$
\frac{\partial \mathcal{L}_{\beta}\{f(t)\}_{(s)}}{\partial s}=\int_{0}^{\infty}(-t \log \beta) \beta^{-s t} f(t) d t=-\log \beta \mathcal{L}_{\beta}\{t f(t)\}_{(s)} .
$$

Taking the partial derivative of equation with respect to $\beta$, we get

$$
\frac{\partial \mathcal{L}_{\beta}\{f(t)\}_{(s)}}{\partial \beta}=\int_{0}^{\infty}\left(-\frac{s t}{\beta}\right) \beta^{-s t} f(t) d t=-\frac{s}{\beta} \mathcal{L}_{\beta}\{\operatorname{tf}(t)\}_{(s)} .
$$

By equations (2.1) and (2.2)

$$
\begin{aligned}
\frac{\partial \mathcal{L}_{\beta}\{f(t)\}_{(s)}}{\partial s} & =\frac{\beta \log \beta}{s} \frac{\partial \mathcal{L}_{\beta}\{f(t)\}_{(s)}}{\partial \beta} \\
& \Rightarrow\left\{s \frac{\partial}{\partial s}-\beta \log \beta \frac{\partial}{\partial \beta}\right\} \mathcal{L}\{f(t)\}_{(s)}=0, \\
& \Rightarrow \mathcal{T} \mathcal{L}\{f(t)\}_{(s)}=0, \text { where } \mathcal{T} \equiv\left\{s \frac{\partial}{\partial s}-\beta \log \beta \frac{\partial}{\partial \beta}\right\} .
\end{aligned}
$$

We can get necessary condition for existence of the Laplace transform for a function of class A by taking limit $\beta \rightarrow e$ and we obtain

$$
\lim _{\beta \rightarrow e} \mathcal{T} \mathcal{L}\{f(t)\}_{(s)}=0 .
$$

\section{Conclusion}

We have discussed and proved that the $\beta$-Laplace transform has some advantage (for having some special properties) over the classical Laplace transform for the functions of class A. This new generalised Laplace transform possesses more powerful tools (special properties) than the Laplace transform, such as shifting of poles, variable s-domain, variable domain of analyticity, and a necessary condition for the existence. 


\section{Acknowledgment}

The author K. S. Nisar expresses his thanks to the Deanship of Scientific Research (DSR), Prince Sattam bin Abdulaziz University, Saudi Arabia for providing facilities and support.

\section{References}

[1] K. Aboodh, The new integral transform "Aboodh transform", Global J. Pure Appl. Math., 9 (2013), 35-43. 1

[2] R. E. Bellman, R. S. Roth, The Laplace transform, World Scientific Publishing Co., Singapore, (1984). 1

[3] P. Chaudhary, P. Chanchal, Y. Khandelwal, Y. Singh, Duality of "some famous integral transform" from the polynomial integral transform, International Journal of Mathematics Trends and Technology, 55 (2018), 345-349. 1

[4] L. Debnath, D. Bhatta, Integral Transform and Their Application, CRC Press, Boca Raton, (2015). 1

[5] G. Doetsch, Handbuch der Laplace-transformation, Band III, Birkhäuser Verlag, Basel-Stuttgart, (1956). 1

[6] G. Doetsch, Introduction to the theory and applications of the Laplace transformation, Springer-Verlag, New York, (1970). 1

[7] T. M. Elzaki, The new integral transform 'Elzaki transform', Global J. Pure Appl. Math., 7 (2011), 57-64. 1

[8] T. M. Elzaki, S. M. Elzaki, On the relationship between Laplace and new integral transform 'Tarig transform', Appl. Math., 36 (2011), 3230-3233. 1

[9] F. Fichard, G. Lyons, Understanding digital signal Processing, Second ed., Prentice Hall PTR, New York, (2004). 1

[10] A. Gaur, G. Agarwal, On ß-Laplace integral transforms and their properties, Int. J. Adv. Sci. Tech., 29 (2020), $1481-1491$. 1

[11] J. C. Jaeger, G. Newstead, An introduction to the Laplace transformation with engineering applications, Third ed., Methuen \& Co., London, (1969). 1

[12] A. Kamal, H. Sedeeg, The new integral transform "Kamal transform", Adv. Theor. Appl. Math., 11 (2016), 451-458. 1, 2.4

[13] Z. H. Khan, W. A. Khan, N-transform properties and applications, NUST J. Eng. Sci., 1 (2008), 127-133.

[14] M. Mohand, A. Mahgoub, The new integral transform "Mahgoub transform", Adv. Theor. Appl. Math., 11 (2016), 391-398.

[15] M. Mohand, A. Mahgoub, The new integral transform "Mohand transform", Adv. Theor. Appl. Math., 12 (2017), $113-120$.

[16] M. Mohand, A. Mahgoub, The new integral transform "Sawi transform", Adv. Theor. Appl. Math., 14 (2019), 81-87. 1

[17] M. Olivi, The Laplace transform in control theory. In: Harmonic Analysis and Rational Approximation, 2006 (2006), 193-209. 1

[18] I. Podlubny, Fractional Differential Equations, Academic Press, San Diego, (1999). 1

[19] H. Saleh, E. Alali, A. Ebaid, Medical applications for the flow of carbon-nanotubes suspended nanofluids in the presence of convective condition using Laplace transform, J. Association Arab Univer. Basic Appl. Sci., 24 (2017), 206-212. 1

[20] J. L. Schiff, The Laplace Transform: Theory and Applications, Springer-Verlag, New York, (1999). 1

[21] S. L. Shaikh, Introducing a new integral transform: Sadik transform, Amer. Int. J. Res. Sci. Tech. Eng. Math., 22 (2018), 100-102. 1

[22] I. N. Sneddon, The use of integral transforms, McGraw-Hill Book Co., New York, (1972). 1

[23] E. J. Watson, Laplace transforms and applications, Van Nostrand Reinhold Co., New York-London, (1981). 1

[24] G. K. Watugala, Sumudu transform: a new integral transform to solve differential equations and control engineering problems, Internat. J. Math. Ed. Sci. Tech., 24 (1993), 35-43. 1

[25] M. Wójcik, M. Szukiewicz, P. Kowalik, The application of the Laplace transform for modelling of gas flow using maple, J. Appl. Comput. Sci. Methods, 6 (2014), 43-53. 1

[26] Z. U. A. Zafar, ZZ-transform method, Int. J. Adv. Eng. Global Tech., 4 (2016), 1605-1610. 1 